\title{
Prognosis and Management in Subsequent Rh Alloimmunized Pregnancies
}

\author{
Andreea DUMITRU ${ }^{a}$, Nicolae GICA ${ }^{a}$,, Radu BOTEZATU ${ }^{a, b}$, Gheorghe PELTECU ${ }^{a, b}$, \\ Mihaela DEMETRIANa, Anca Maria PANAITESCU ${ }^{a, b}$ \\ a"Filantropia" Clinical Hospital, Bucharest, Romania \\ b"Carol Davila" University of Medicine and Pharmacy, Bucharest, Romania
}

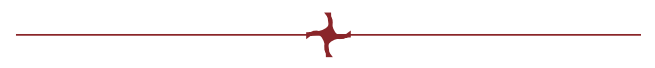

\begin{abstract}
Background: RhD alloimmunization remains a severe problem worldwide, but its management has been
revolutionized by two important discoveries: the possibility to establish fetal Rh genotype non-invasively by
using a maternal blood sample, and using of Doppler velocimetry to monitor early signs of affected fetuses.
Materials and methods: We performed a literature review by searching PubMed for relevant information
about diagnosis, prognosis, and management of secondary affected Rh alloimmunized pregnancies.
Results: The risk to develop fetal anemia and hydrops seems to increase with increasing concentrations
of Rh antibodies, and studies show it is higher for subsequent pregnancies. Individuals presenting the DEL
phenotype with the types 1, 2 or 3 can be considered RhD positive and anti-D immune globulin is not indicated.
Discussions: Medical algorithm involves previous pregnancy history together with serum parameters.
Follow-up in a department of maternal fetal medicine is desired and encouraged in these cases. Depending on
the severity and woman's previous pregnancy history, especially condition prior to 24 weeks of gestation, several
therapies such as plasmaphereses, intravenous immune globulin or intrauterine transfusions can be conducted.
Intrauterine transfusions have a better prognosis when performed earlier and on fetuses without hydrops.
Conclusions: Although the incidence of hemolytic disease of the fetus and newborn has decreased and
is no longer a major cause of perinatal mortality, vigilance is still required. There is a strong argument for
reunite the management of these cases in dedicated maternal fetal medicine centers that perform invasive
procedures in order to improve knowledge, gain skills and enhance clinical management.
Keywords: alloimmunization, D antigen, anti Rh antibody, pregnancy,
fetal anemia, hydrops fetalis.
\end{abstract}

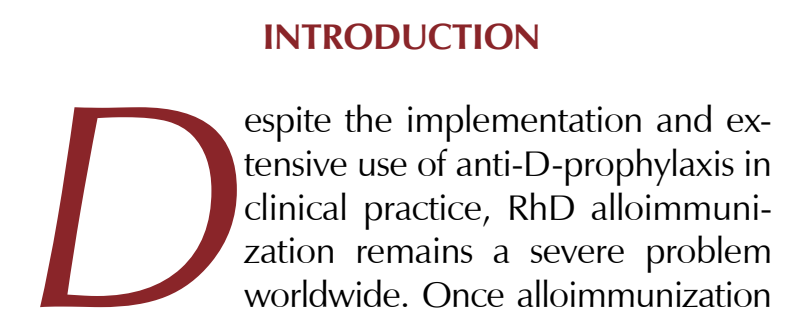

has occurred it can cause hemolytic disease of the fetus and newborn (HDFN) and hydrops fetalis and this risk seems to increase with increasing concentrations of antibodies. Without treatment, it can even result into intrauterine fetal death. The management of RhD alloimmunization has been revolutionized by two important discoveries: firstly, the possibility to establish fetal Rh genotype non-invasively by using a maternal blood sample,

\footnotetext{
Address for correspondence:

Nicolae Gica

Email: gica.nicolae@umfcd.ro
} 
and secondly, the use of velocimetry of the fetal middle cerebral artery to detect early sign of fetal anemia.

Particularly, these cases are usually addressed to a maternal-fetal medicine specialist with appropriate experience who can perform invasive diagnostic and therapeutic procedures that may be needed for the best outcome of the pregnancy.

\section{Pathophysiology}

$\mathrm{D}$ antigen is present only on the surface of red blood cells (RBC) and it starts to be expressed on the cell membrane by the 38 days of gestation. As a result of maternal immune system exposure to RhD positive RBCs, maternal D alloimmunization starts developing. Further, anti-D circulating antibodies (Ig G) cross the placenta and opsonize the fetal red blood cells, which are phagocytized by macrophages in the fetal spleen, therefore producing fetal anemia $(1,2)$.

Several events may cause maternal alloimmunization, including the delivery, induced or spontaneous abortion, ectopic pregnancy, invasive fetal-maternal procedures, external cephalic version, antenatal hemorrhage, abruption placenta and even maternal trauma (3-6).

As the current literature shows, the volume of blood infused capable to produce an immune response to $\mathrm{RhD}$ negative individuals is $0,45 \mathrm{~mL}$ in about $80 \%$ of persons, with some responding to volumes of $0,5 \mathrm{~mL}$, but with known exceptions where as little as $0,1 \mathrm{~mL}$ was enough to immunize some individuals. The response develops slowly and can be detected serologically around 15 weeks after exposure; therefore, earlier serological detection provides no benefit (7).

\section{The DEL phenotype}

The Rh system provides different phenotypes and obstetricians should be aware of them to provide suitable maternal and fetal care. Usually, the standard nomenclature for a pregnant woman blood type is the ABO type and either "Rh positive" or "Rh negative", which is suggestive to describe an individual who has or does not have the $\mathrm{D}$ antigen on the red blood cells. But, besides $\mathrm{D}$ antigen there are other frequent antigens as d, C, c, E, e, and $\mathrm{G}$ that can explain the phenomenon when an RhD-negative mother may have received prophylactic anti-D immune globulin and this did not prevent alloimmunization to other Rh antigens (e.g., c, C, E, e).
Zygosity is another characteristic of the Rh system. Studies reveal that approximately $40 \%$ of $\mathrm{Rh} \mathrm{D}$ positive individuals are homozygous for the $\mathrm{D}$ antigen (DD), while the remaining ones are heterozygous (Dd) (8). Also, it is important to mention that there are also $\mathrm{D}$ variants. For the white population, the basis of Rh D negative phenotype is the absence of $\mathrm{D}$ gene (RHD), while in other racial or ethnic groups the gene might be present but not translated or expressed (9).

A special discussion is about the "weak D" or "DEL variant" that may alter the usual treatment algorithm. Depending on the reactive employed by a laboratory, some individuals undergoing D typing will reveal a "weak D" expression on initial testing. When testing the red cells with an anti-D reactive, they have no, or less than, a $2+$ reaction, but when adding antihuman globulin, a moderate to strong reaction occurs. The explanation for this resides in either a smaller number of intact $D$ antigens on the surface of the red cells ("weak D type") or D antigens that are missing a portion - epitopes. Only $0.2-1 \%$ of the white population inherited this variant and if individuals present the types 1, 2 or 3, they can be considered RhD positive, and anti-D immune globulin is not indicated $(10,11)$.

\section{Management algorithm}

The presence of maternal anti-D antibody should be evaluated at the first prenatal visit, together with the blood type of both parents.

The management of Rhesus alloimmunization is different whether we talk about first affected pregnancy or subsequent ones, because the mother's anti-D titer is usually low at the beginning of her first affected pregnancy and fetal anemia may not develop or develops in the late second or third trimester. On the other hand, for future affected pregnancies the fetal hemolytic syndrome is usually more severe and appears earlier during the gestation. For this reason, frequent serial dosing of maternal antibodies and, when necessary, serial Doppler assessment of the peak systolic velocity in the middle cerebral artery is useful during the first-time sensitized pregnancy, while for secondary or more-time sensitized pregnancies, maternal titers are no longer predictive of risk, making Doppler ultrasonography be of extreme importance in predicting fetal anemia (12).

After a previous pregnancy affected by Rh (D) alloimmunization, gradual steps should be fol- 
lowed in maternal-fetal care. History regarding the previous perinatal outcome is important (together with gestational age of the first signs of fetal anemia) along with the baseline level of anti $D$ antibody. Secondly, the paternal $\mathrm{Rh}(\mathrm{D})$ type and zygosity should be documented, if not done previously or if the partner has changed. From this step, two possibilities arise:

- if the paternal $\mathrm{Rh}(\mathrm{D})$ is negative, the fetus will not be at risk and after the documentation of this analysis in the medical record of the pregnant woman, she will receive routine prenatal care or,

- if the paternal $\mathrm{Rh}(\mathrm{D})$ is positive and homozygous, the fetus will surely be RhD positive, while if the paternal $\mathrm{Rh}(\mathrm{D})$ is positive and heterozygous, we can obtain the fetal Rh by the use of free fetal DNA testing and follow up a medical conduct accordingly.

The worst-case scenario with the RhD-positive fetus involves considerations about fetal anemia or hydrops earlier than 24 weeks of gestation or anti D-titer higher than 1:1028 (13).

1. In case none of these appeared before 24 weeks gestation, the suitable way to follow potential fetal injury is by starting weekly middle-cerebral artery (MCA) Doppler ultrasound at 16 to 18 weeks of gestation. Proper technique is important, so the measurement is counseled to be obtained when the fetus is in a quiet behavioral state. Because the value of MCA-PSV increases during gestation, there are tools for conversion into MoMs like the one from https://www.perinatology.com.calculators. The reference point for the MCA peak systolic velocity (MCA-PSV) is 1.5 multiples of the median (MoMs) recommended by both societies of perinatology and maternal fetal medicine. Under or equal to this value, the antenatal testing can begin at 32 weeks of gestation and delivery is recommended to be hasted between 37 and 38 weeks. A peak systolic value greater than 1.5 MoMS involves invasive procedures performed by a maternal fetal specialist with expertise in this field to check the fetal hemoglobin level by cordocentesis, a not to ignore step, bearing in mind that MCA-PSV is not a definitive marker for fetal anemia and false positive results may occur. After carefully informing the patient about risks and benefits, the final decision for intrauterine transfusions is made when either the fetal hemoglobin is lower than $2 \mathrm{SD}$ below the mean for gestational age or the hematocrit volume is under $30 \%$. Otherwise, the hemoglobin level is checked again after two weeks, and follow-up is continued accordingly (14).

2. In case when one or the two conditions appeared before 24 weeks, plasmaphereses or intravenous immune globulin (IVIG) can be administered to prevent fetal injury and then continue with the assessment of MCA-PSV as described before.

\section{Prevention of future affected fetuses}

Prevention of hemolytic disease of the fetus and newborn (HDFN) is rarely attempted because of the involved costs and complexities (family good understanding of the diagnosis, environmental factors - access to high specialized medical care, but also because in most cases HDFN can be treated).

Possibilities include in vitro fertilization with preimplantation genetic testing. If the biologic father is identified as heterozygous for RhD, only the RhD negative embryos are considered for transfer (15). Also, in countries where the national legislation permits, a gestational carrier can be used in case the mother is RhD negative and the father is RhD positive homozygous to prevent alloimmunization. A third way, also depending on the national legislation and rules, can be the use of donor sperm from a blood type compatible with the mother, an RhD negative person too.

\section{DISCUSSIONS}

A hen fetal anemia presents very early in gestation, the reported survival rates of fetuses with and without hydrops are still dramatically different (16). Improved perinatal outcome is directly related to reversal of hydrops due to intrauterine treatment. On the other side, in cases where the hydrops did not reverse, the survival rate was only $39 \%$, but with mentions in the current literature of a proportion of irreversible cases despite successful correction of fetal anemia (17).

In the management of a Rh alloimmunized pregnancy, obstetricians should be aware of the existence of particular D phenotypes that may change the standard conduct. A particular variant, the "weak D" or "DEL variant", involves whether a smaller number of intact $D$ antigens on the surface of the red cells ("weak D type") or D antigens that are missing a portion- epitopes, and if sub- 
types 1, 2 or 3 are present, these antibodies can be considered $\mathrm{RhD}$ positive and anti-D immune globulin is not indicated.

For subsequently affected pregnancies, the fetal hemolytic syndrome is usually more severe and appears earlier during gestation. For this reason, during the first-time sensitized pregnancy is useful the frequent serial dosing of maternal antibodies and, when necessary, serial Doppler assessment of the peak systolic velocity in the middle cerebral artery, while for secondary or more-time sensitized pregnancies, maternal antibody level is no longer predictive of risk, making Doppler ultrasonography be of extreme importance in predicting fetal anemia (12).

\section{CONCLUSIONS}

Progr rognosis of subsequent $\mathrm{Rh}$ alloimmunized pregnancies depends on the moment of establishing the diagnosis of fetal injury, personal maternal history regarding previous pregnancy/pregnancies and environmental factors, considering the possibility to access maternal fetal specialists and highly specialized care.

For individuals affected by Rh alloimmunization, a desired pregnancy plan includes frequent monitoring in a maternal fetal care unit by an experienced specialist and a management algorithm concordant with the latest clinical guidelines on the topic.

Perinatal outcome is directly related to the reversal of fetal hydrops due to intrauterine transfusions, showing an improvement for fetuses with both anemia and hydrops.

The rate of survival for fetuses diagnosed with anemia has considerably improved since the use of intrauterine transfusions, but vigilance is required.

Conflicts of interest: none declared.

Financial support: none declared.

Authors' contributions: All authors have contributed equally.

All authors read and approved the final version of the manuscript.

\section{$\boldsymbol{R}_{\text {EFERENCES }}$}

1. Bergström $H$, Nilsson LA, Nilsson $L$, Ryttinger L. Demonstration of Rh antigens in a 38-day-old fetus.

Am J Obstet Gynecol 1967;99:130.

2. Kumpel BM. On the immunologic basis of Rh immune globulin (anti-D) prophylaxis. Transfusion 2006;46:1652.

3. Horvath S, Tsao P, Huang ZY, et al. The concentration of fetal red blood cells in first-trimester pregnant women undergoing uterine aspiration is below the calculated threshold for $\mathrm{Rh}$ sensitization. Contraception 2020;102:101.

4. Meleti D, De Oliveira LG, Araujo Júnior E, et al. Evaluation of passage of fetal erythrocytes into maternal circulation after invasive obstetric procedures. J Obstet Gynaecol Res 2013;39:1374.

5. Lipitz S, Achiron R, Horoshovski D, et al. Fetomaternal haemorrhage discovered after trauma and treated by fetal intravascular transfusion.

Eur J Obstet Gynecol Reprod Biol 1997;71:21.

6. Boucher M, Marquette GP, Varin J, et al. Fetomaternal hemorrhage during external cephalic version.

Obstet Gynecol 2008;112:79.

7. Kumpel BM. On the mechanism of tolerance to the Rh D antigen mediated by passive anti-D ( $R h$ D prophylaxis).
Immunol Lett 2002;82:67.

8. Moise $\mathrm{KJ} \mathrm{Jr}$. $\mathrm{RhD}$ alloimmunization in pregnancy: Overview, Aug 2021. Last accessed: 8 September 2021 at https://www.uptodate.com/contents/ rhd-alloimmunization-in-pregnancyoverview? search $=\mathrm{rh} \% 20$ alloimmunization\&source $=$ search $\_$result\& selectedTitle $=1 \sim 150 \&$ usage type $=$ default\&display_rank $=1$.

9. Wagner FF, Gassner C, Müller TH, et al. Molecular basis of weak D phenotypes. Blood 1999;93:385.

10. Garratty G. Do we need to be more concerned about weak D antigens? Transfusion 2005;45:1547.

11. Sandler SG, Flegel WA, Westhoff CM, et al. It's time to phase in RHD genotyping for patients with a serologic weak D phenotype. College of American Pathologists Transfusion Medicine Resource Committee Work Group.

Transfusion 2015;55:680.

12. Moise KJ. Management of Rhesus Alloimmunization in Pregnancy. Obstetrics \& Gynecology 2008;112:164-176. doi:10.1097/aog.0b013e31817d453c.

13. Mari G, Norton ME, et al. Society for Maternal-Fetal Medicine (SMFM) Clinical
Guideline \#8: the fetus at risk for anemia--diagnosis and management. Am J Obstet Gynecol 2015;212:697.

14. Phung TV, Houfflin-Debarge V, Ramdane N, Ghesquière L, Delsalle A, Coulon $\mathrm{C}$ et al. Maternal red blood cell alloimmunization requiring intrauterine transfusion: a comparative study on management and outcome depending on the type of antibody. Transfusion 2018;58:1199-1205. doi:10.1111/trf.14542.

15. Seeho SK, Burton G, Leigh D, et al. The role of preimplantation genetic diagnosis in the management of severe rhesus alloimmunization: first unaffected pregnancy: case report. Hum Reprod 2005;20:697.

16. Klumper FJ, van Kamp IL, Vandenbussche FP, Meerman RH, Oepkes D, Scherjon SA, et al. Benefits and risks of fetal red-cell transfusion after 32 weeks gestation. Eur J Obstet Gynecol Reprod Biol 2000;92: 91-96.

17. van Kamp IL, Klumper FJ, Bakkum RS, et al. The severity of immune fetal hydrops is predictive of fetal outcome after intrauterine treatment. Am J Obstet Gynecol 2001;185: 668-673. 